\title{
Differential Regulation of NMDAR1 mRNA and Protein by Estradiol in the Rat Hippocampus
}

\author{
Adam H. Gazzaley, ${ }^{1}$ Nancy G. Weiland, ${ }^{3}$ Bruce S. McEwen, ${ }^{3}$ and John H. Morrison ${ }^{1,2}$ \\ ${ }^{1}$ Laboratories for Neurobiology of Aging, Fishberg Research Center for Neurobiology, New York, New York 10029-6574, \\ 2 Department of Geriatrics and Adult Development, The Mount Sinai School of Medicine, New York, New York 10029, and \\ 3Laboratory of Neuroendocrinology, Rockefeller University, New York, New York 10021
}

\begin{abstract}
Estradiol treatment increases the number of NMDA receptor binding sites, and changes evoked synaptic currents in a manner consistent with a steroid-induced functional enhancement of NMDA receptors in rat hippocampus. In this study, we investigate the cellular mechanisms of estradiol-induced NMDA receptor regulation at the protein and $\mathrm{mRNA}$ levels in ovariectomized rats treated with ovarian steroids using immunocytochemical and in situ hybridization techniques. Confocal laser scanning microscopy was used to quantify alterations in immunofluorescence intensity levels of NMDAR1 subunit proteins within neuronal somata and dendrites of discrete hippocampal fields, whereas in parallel, in situ hybridization was used to examine NMDAR1 mRNA levels in corresponding hippocampal regions. The data indicate that estradiol treatment in ovariectomized rats significantly increases immunofluorescence inten-
\end{abstract}

sity levels in comparison with nonsteroid treated ovariectomized rats within the somata and dendrites of CA1 pyramidal cells and, to a lesser extent, within the granule cell somata of the dentate gyrus. In contrast, such alterations in immunofluorescence intensity occur without concomitant changes in mRNA hybridization levels. Thus, these data suggest that estradiol modulates NMDA receptor function via posttranscriptional regulation of the NMDAR1 subunit protein. The increase in immunofluorescence intensity may reflect an increase in the concentration of the subunit protein, which could account for estrogen-induced changes in pharmacological and physiological properties of the NMDA receptor.

Key words: excitatory amino acid receptors; NMDAR1; immunocytochemistry; in situ hybridization; estrogen; CA1; confocal microscopy
Ovarian steroids affect brain regions and behaviors that are not directly associated with reproductive functions (McEwen et al., 1995). However, the mechanisms by which these effects are produced have not yet been determined. NMDA receptors (NMDARs), a subtype of ionotropic glutamate receptors (Moriyoshi et al., 1991), are implicated as mediators of effects of estradiol on morphological plasticity and related physiological and cognitive processes in the brain. For example, in the CA1 field of rat hippocampus, estradiol treatment after ovariectomy increases dendritic spine density (Gould et al., 1990) and synapses (Woolley and McEwen, 1992) on pyramidal cells via a mechanism dependent on NMDAR activation (Woolley and McEwen, 1994). Additionally, intracellular recordings reveal that estradiol treatment increases the duration of EPSPs in a subpopulation of CA1 neurons, an alteration suggestive of an increased NMDAR contribution (Wong and Moss, 1992). Less direct evidence of NMDAR involvement in estrogen-induced neural modifications includes estrogen's role in facilitating seizure induction (Terasawa and Timiras, 1968; Backstrom, 1976; Buterbaugh and Hudson, 1991) and memory and learning enhancement (Philips and Sherwin, 1992a,b; Luine, 1994; Singh et al., 1994) in both humans and experimental animals. This connection is based on the NMDAR's important role in seizure-triggering mechanisms (Gilbert, 1988;

Received June 17, 1996; revised July 25, 1996; accepted Aug. 16, 1996.

This work was supported by the Charles A. Dana Foundation and National Institutes of Health Grants AG-06647 (J.H.M.), NS-30105 (N.G.W.), and NS-07080 (B.S.M.). We thank Dr. George Huntley for helpful comments on this manuscript. Correspondence should be addressed to Dr. John H. Morrison, Fishberg Research Center for Neurobiology, The Mount Sinai School of Medicine, P.O. Box 1065, One Gustave L. Levy Place, New York, NY 10029-6574.

Copyright (C) 1996 Society for Neuroscience $0270-6474 / 96 / 166830-09 \$ 05.00 / 0$
Sato et al., 1989) and memory and learning (Morris et al., 1986; Bliss and Collingridge, 1993).

Few studies have directly addressed the mechanisms underlying estrogen regulation of NMDARs. Autoradiographic analysis has revealed that NMDAR agonist binding sites are increased in the dendritic layer of CA1 in response to estradiol treatment in ovariectomized rats (Weiland, 1992). However, because an alteration in receptor stoichiometry or regulation by modulatory ligands can alter ligand binding, these results do not determine whether steroidal treatment induced an increase in NMDA receptor subunit protein levels in these neurons. Additionally, no studies have investigated the effect of ovarian hormone treatment on NMDAR subunit mRNA levels in hippocampal neurons. Therefore, the focus of this study is to elucidate the cellular mechanisms by which hippocampal NMDARs are regulated by ovarian steroids. Through the use of quantitative confocal laser scanning microscopy (CLSM), we have recently demonstrated that the immunocytochemically localized cytoplasmic pool of the NMDAR subunit 1 (NMDAR1), an obligatory subunit of the NMDAR complex (Monyer et al., 1992; Nakanishi, 1992), is modifiable in hippocampal neurons during aging (Gazzaley et al., 1996a) and in response to deafferentation (Gazzaley et al., 1996b). These data suggest that alterations in cytoplasmic receptor protein levels is a component of the neuronal response strategy to various conditions. To investigate the role of estradiol and progesterone in regulating NMDARs at the protein level, we performed a CLSM evaluation of NMDAR1 immunofluorescence intensity in the somata and dendrites of the dentate gyrus and CA1 and CA3 hippocampal fields of ovariectomized rats and ovariectomized rats treated with estradiol and estradiol plus pro- 
gesterone. A CLSM analysis was selected for this study, because it is performed on structurally intact tissue and permitted the evaluation of discrete cell groups and intracellular compartments, which would not have been possible by biochemical methods such as a Western blot analysis of homogenized tissue extracts. Additionally, to determine whether changes observed at the protein level reflect changes at the level of gene transcription, we examined NMDAR1 mRNA levels by quantitative in situ hybridization analysis in the same hippocampal regions.

\section{MATERIALS AND METHODS}

Animal and tissue processing. Forty-three young adult Sprague Dawley rats (Charles River, Wilmington, MA), weighing $\sim 250 \mathrm{gm}$, were maintained in a temperature- and light-controlled environment with a light (14 $\mathrm{hr}$ )/dark $(10 \mathrm{hr})$ cycle (lights on at $0500 \mathrm{hr})$. Animals were treated in accordance with the principles and procedures of the National Institutes of Health Guide for the Care and Use of Laboratory Animals, and all surgeries were performed under Metofane anesthesia. Sixteen rats were used in the immunocytochemical analysis. All 16 rats were ovariectomized for 1 week, at which time 10 rats received SILASTIC capsules containing $180 \mu \mathrm{g}$ of $17-\beta$ estradiol/sesame oil, and the remaining 6 were sham operated. Two days later at $1000 \mathrm{hr}$, five of the estradiol-treated animals were injected subcutaneously with progesterone $(1 \mathrm{mg}$ in $0.3 \mathrm{ml}$ $\mathrm{oil} / \mathrm{rat}$ ), and all remaining animals were injected with oil. This procedure resulted in the following groups of ovariectomized rats: six sham plus oil (OVX), five estradiol plus oil $(\mathrm{OVX}+\mathrm{E})$, and five estradiol plus progesterone $(\mathrm{OVX}+\mathrm{E}+\mathrm{P})$. Five hours after progesterone or oil injection $(1500$ $\mathrm{hr}$ ), the animals were deeply anesthetized with Metophane and transcardially perfused with cold $1 \%$ paraformaldehyde in $0.1 \mathrm{M}$ PBS followed by cold $4 \%$ paraformaldehyde in PBS. The brains were removed, post-fixed in $4 \%$ paraformaldehyde, and sectioned at $40 \mu \mathrm{m}$ on a vibratome (OTS 3000, Electron Microscopy Sciences, Fort Washington, PA). The sections were stored in PBS with $0.1 \%$ sodium azide at $4^{\circ} \mathrm{C}$.

Twenty-seven rats were used for the in situ hybridization study. All rats were ovariectomized for 1 week and then adrenalectomized. Eighteen rats were then treated with SILASTIC capsules containing $180 \mu \mathrm{g}$ of $17-\beta$ estradiol/sesame oil, whereas the other nine were sham operated. Two days later at $1000 \mathrm{hr}$, the animals were either injected subcutaneously with progesterone $(1 \mathrm{mg}$. in $0.3 \mathrm{ml} \mathrm{oil} / \mathrm{rat})$ or oil, resulting in the following groups of ovariectomized rats: nine sham plus oil (OVX), nine estradiol plus oil $(\mathrm{OVX}+\mathrm{E})$, and nine estradiol plus progesterone $(\mathrm{OVX}+\mathrm{E}+\mathrm{P})$. The animals were killed by decapitation $5 \mathrm{hr}$ after progesterone or oil injection $(1500 \mathrm{hr})$, and the brains were removed, frozen on dry ice, and stored at $-70^{\circ} \mathrm{C}$.

In situ hybridization. In situ hybridization was performed using previously published sequences of oligonucleotides (Oligos Etc., Wilsonville, OR) complementary to rat cDNA encoding subunit residues between putative transmembrane domains I and II, encoding amino acids 566580, and recognizing all published splice variants (Monyer et al., 1992). A search of the GenBank database indicated that there is no significant homology among any of these sequences and known mammalian gene sequences. Oligonucleotides were $3^{\prime}$ end-labeled with terminal transferase (Boehringer Mannheim, Indianapolis, IN) using a 2:1 molar ratio of $\left[\alpha^{-35} \mathrm{~S}\right]$ dATP:cDNA (1200-1400 Ci/mmol; New England Nuclear, Boston, MA). Unincorporated nucleotides were removed using NucTrap push columns (Stratagene Cloning Systems, La Jolla, CA). Hybridization was performed as described previously, (Orchinik et al., 1994). Briefly, sections were fixed in $4 \%$ paraformaldehyde, acetylated, hybridized with saturating concentrations of labeled oligonucleotides overnight at $42^{\circ} \mathrm{C}$, washed to a final stringency of $0.1 \times \operatorname{SSC}(1 \times=0.15 \mathrm{M} \mathrm{NaCl}$ and 0.015 M sodium citrate) at $55^{\circ} \mathrm{C}$ for $1 \mathrm{hr}$, dehydrated, and exposed to Hyperfilm$\beta \max$ (Amersham, Arlington Heights, IL) for $24 \mathrm{hr}$.

Quantitative in situ hybridization analysis. The films were analyzed by measuring the optical density of specific regions of the hippocampus using an automated paint function that covered the region of interest (Imaging Research, St. Catherines, Ontario, Canada). Bilateral measurements were taken from four sections per animal, nine animals per group in the principal cell layer of CA1, CA3, and the suprapyramidal layer of the dentate gyrus. Background was measured from the corpus callosum and subtracted from the total optical density.

Immunocytochemistry. Three nonadjacent sections from the rostral hippocampus of each rat were incubated with monoclonal antibodies to both the NMDAR1 subunit (54.1) (Siegel et al., 1994) and microtubule- associated protein 2 (MAP2) (Huber and Matus, 1984) at a concentration of 4.6 and $2.5 \mu \mathrm{g} / \mathrm{ml}$, respectively, in PBS for $48 \mathrm{hr}$. Sections were then washed three times in PBS, transferred to biotinylated anti-mouse IgG (Vector Laboratories, Burlingame, CA) for $2 \mathrm{hr}$, washed again in PBS, and transferred to FITC-conjugated avidin (Vector Laboratories) for 1 hr. Sections were then mounted and coverslipped with Vectashield (Vector Laboratories) to reduce fluorescence quenching.

CLSM and quantitative immunocytochemical evaluation. Quantitative CLSM analysis was performed on three sections from each rat brain, for each of the two antibodies, with a Zeiss LSM 410 inverted confocal microscope (Thornwood, NY). The investigator was blinded throughout the evaluation as to which sections were from which experimental group. The quantitative analysis performed in this study was adapted from a previous study that quantified relative differences in immunofluorescence intensity levels (Gazzaley et al., 1996b). The confocal parameters were established at the beginning of the study and remained constant throughout. An Argon/Krypton laser was used to excite FITC at $488 \mathrm{~nm}$. A $90 \%$ neutral density filter was used to attenuate the light, and a confocal aperture pinhole setting of 17 was set digitally. The image was visualized with a Zeiss Plan-Neofluar $63 \times / 1.25$ NA oil immersion objective. For each antibody, a contrast/brightness setting was selected that yielded a high-resolution image for both bright and dim sections without exceeding a maximal pixel intensity of 255 . For the NMDAR1 analysis, a separate contrast/brightness setting was established for somata and dendrites because of a considerable intensity difference between them that prevented the use of a single setting. All of the settings were kept constant throughout the analysis to yield unbiased measurements for each set of comparisons.

For the study of somatic immunofluorescence intensity, six fields were randomly selected on each section within a centrally located region of the suprapyramidal blade of the dentate gyrus granule cell layer and the middle portion of the CA1 and CA3 pyramidal cell layer. The dendritic immunofluorescence intensity study consisted of six randomly selected dendritic fields, on each section, from the middle molecular layer of the dentate gyrus and the stratum radiatum of CA1 adjacent to the somatic regions analyzed in the somatic immunofluorescence analysis. All dendritic fields scanned were the same size $\left(3832 \mu \mathrm{m}^{2}\right)$ and all were selected at a distance of $\sim 70 \mu \mathrm{m}$ from the principal cell layers. Each field was scanned only once, to reduce fluorescence quenching, and at the same predetermined $z$ axis distance from the surface of the section. Scanning was performed with a two line average for a total scan time of $4.52 \mathrm{sec}$ and an electronic zoom factor of 3.28 , which increased the resolution to $0.0081 \mu \mathrm{m} /$ pixel. Each digitized image consisted of a $512 \times 512 \times$ eight bit pixel array, in which every pixel was assigned a gray level intensity value ranging from 0 to 255 . An image-analysis program (Zeiss) was used to determine the average pixel intensity of each field. To remove the negative contribution of unlabeled portions of the field to the average field intensity (i.e., nuclei and unlabeled regions between soma and dendrites), a photometric offset was used to establish a pixel intensity threshold below which a pixel would have no contribution to the average pixel intensity of the field. The threshold was set by viewing the image at a display magnification of $2 \times$ and manually increasing the thresholding value until a blue display, designating the thresholded area, completely occupied the unstained nuclei and abutted somatic and dendritic profiles (Fig. 1). Thus, the average pixel intensity of the portion of the field above threshold represents the immunofluorescence intensity within either the dendritic segments or the somata of the principal cell layers.

Data analysis. For the CLSM immunocytochemical analysis, an intensity value was computed for each animal in each of the three regions by determining the mean of the 24 individual field values $(6$ field values obtained from each of three sections). For both the immunocytochemical and in situ hybridization analyses, a mean value for each group was obtained from the individual values determined for each animal, in CA1, CA3, and the dentate gyrus. Percent difference was determined by comparing the steroid-treated ovariectomized rats with the nontreated ovariectomized rats [i.e., $((\mathrm{OVX}+\mathrm{E})-(\mathrm{OVX})) /(\mathrm{OVX})$ or $((\mathrm{OVX}+\mathrm{E}+\mathrm{P})-$ $(\mathrm{OVX})) /(\mathrm{OVX})]$. All the data were analyzed with a one-way ANOVA, at a significance level of $<0.05$, and a Sheffe's post hoc test.

\section{RESULTS}

\section{NMDAR1: somatic immunofluorescence intensity}

NMDAR1 immunolabeling was evaluated in three groups of rats: ovariectomized rats (OVX) and ovariectomized rats that had been treated with either estradiol $(\mathrm{OVX}+\mathrm{E})$ or estradiol plus 

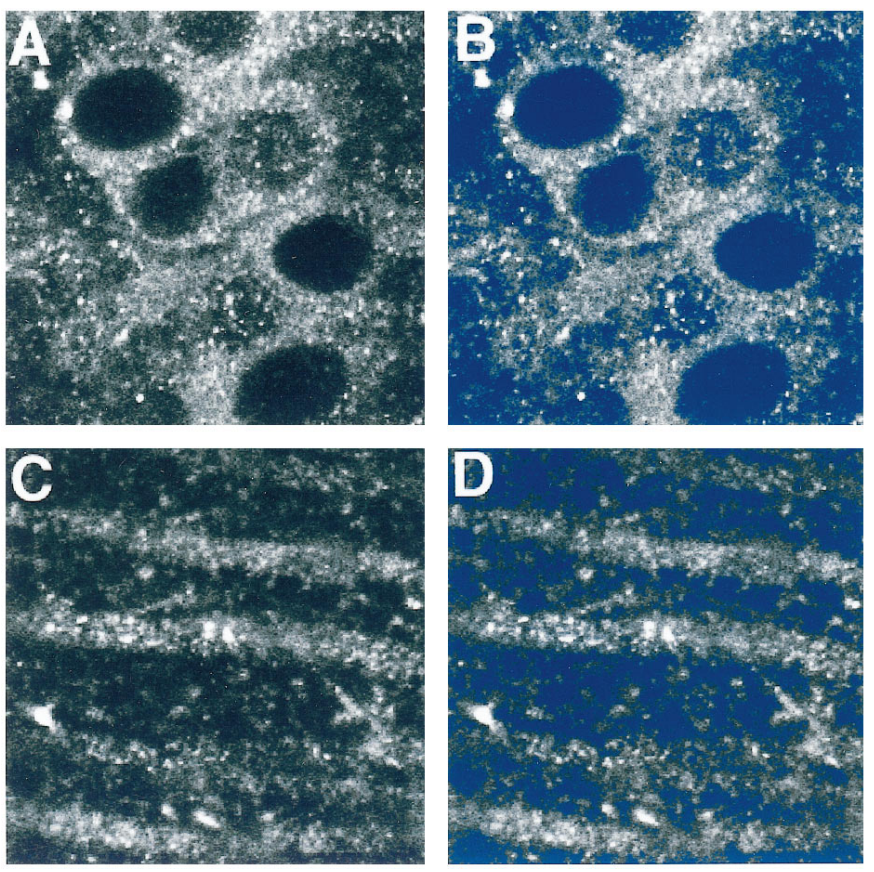

Figure 1. CLSM images of NMDAR1 immunolabeled CA1 pyramidal cell somata $(A, B)$ and dendrites $(B, C)$ before $(A, C)$ and after $(B, D)$ intensity thresholding, as indicated by the blue color overlay. After the thresholding procedure, all unlabeled portions of the field (in blue) have no contribution to the average intensity of the field.

progesterone $(\mathrm{OVX}+\mathrm{E}+\mathrm{P})$. This steroid treatment paradigm has been used previously by Weiland and Orchinik (1995) and results in serum estrogen levels of $\sim 20 \mathrm{pg} / \mathrm{ml}$ and progesterone levels of $60 \mathrm{ng} / \mathrm{ml}$, which mimic preovulatory hormone levels. Nonovariectomized rats were not used in this study, because estrogen levels fluctuate throughout the estrous cycle. In animals from all three groups, NMDAR1 immunofluorescence was present within the cell bodies and dendrites of the hippocampal principal neurons, a general pattern consistent with earlier descriptions of NMDAR1 immunolabeling in the male rat hippocampus (Petralia et al., 1994). Confocal images of pyramidal cells within the CA1 and CA3 subfields and granule cells of the dentate gyrus revealed a patchy intracellular distribution of immunofluorescence throughout the somatic and dendritic cytoplasm, whereas nuclei contained no labeling (see Figs. 2, 3). There were no observable qualitative differences in overall distribution and intracellular pattern of immunolabeling between OVX rats and steroid-treated ovariectomized rats.

Immunofluorescence intensity levels of the cytoplasmic pool of receptors within the somata of the CA1 and CA3 pyramidal cell layers and the dentate gyrus granule cell layer were obtained by CLSM quantitative analysis. All statistical comparisons were made among the three groups, within a given hippocampal subfield. In the CA1 subfield of either the OVX $+\mathrm{E}$ or $\mathrm{OVX}+\mathrm{E}+\mathrm{P}$ group, quantitative data revealed a significant intensity increase within the pyramidal cell somata of $52.2 \%$ in comparison with the OVX group (Fig. $2 a-c$; see also Fig. $4 a$ ). No difference in intensity levels was evident between the two steroid-treatment groups.

In the dentate gyrus, a smaller but statistically significant intensity increase was observed within the somata of the granule cells in $\mathrm{OVX}+\mathrm{E}(31.3 \%)$ and $\mathrm{OVX}+\mathrm{E}+\mathrm{P}(33.5 \%)$ rats relative to the OVX rats (Figs. $2 d-f$; Fig. $4 a$ ). This intensity increase was of a significantly lower magnitude than that recorded in the CA1 field, as determined by a statistical comparison of the CA1/DG ratio among the three groups, which revealed a significant increase in the ratio in the steroid-treatment groups as compared with the OVX group. As in the CA1 subfield, there was no significant difference between the $\mathrm{OVX}+\mathrm{E}$ and the $\mathrm{OVX}+\mathrm{E}+\mathrm{P}$ groups.

Quantitative analysis of the somata of the CA3 pyramidal cells revealed no difference in immunofluorescence intensity levels among the three groups (Figs. $2 g-i, 4 a$ ).

\section{NMDAR1: dendritic immunofluorescence intensity}

To investigate the cytoplasmic pool of the NMDAR1 subunit protein within the dendrites of the two regions where a somatic intensity increase was observed, we analyzed fields of dendritic segments in the CA1 stratum radiatum and the dentate gyrus molecular layer. Quantitative analysis revealed a statistically significant increase in intensity within the dendritic segments of the CA1 field when comparing both the OVX+E and the $\mathrm{OVX}+\mathrm{E}+\mathrm{P}$ groups with the OVX group (35 and 32\%, respectively) (Figs. $3 a-c, 4 b)$. There was no significant intensity difference between the two steroid-treated groups in the CA1 dendrites. Analysis of the dentate gyrus dendritic fields revealed no significant difference among the three groups, but there was a trend toward an increase in both steroid-treated groups compared with the OVX group (Fig. 4b).

\section{MAP2: somatic immunofluorescence intensity}

To access whether the NMDAR1 intensity change may have been the result of a more general increase in protein production, an identical quantitative analysis was performed after immunocytochemical staining with a monoclonal antibody to MAP2. MAP2 was selected because it is localized specifically within the soma and dendrites of all principal cells in the hippocampus, and there are no reports of estradiol-induced changes in MAP2 cytoplasmic concentration. Quantitative and qualitative analysis revealed no significant differences in immunofluorescence intensity or distribution among the three groups in the somata of any hippocampal field analyzed (Figs. 5a-i, 6).

\section{NMDAR1 mRNA labeling}

To determine whether a change in immunofluorescence intensity levels of the NMDAR1 protein corresponded to an alteration in gene transcription, in situ hybridization analysis of NMDAR1 mRNA levels was performed. Hybridization signal was localized within the somata of the dentate gyrus granule cells and the pyramidal cells of the CA fields (Fig. 7), and no overt differences were observed in the overall distribution of hippocampal mRNA labeling in comparison with that described previously in the rat brain (Moriyoshi et al., 1991). Densitometric analysis of the films determined that there was no significant difference in silver grain intensity in the principal cell layers of CA1, CA3, and the dentate gyrus across the three groups (Fig. 8).

\section{DISCUSSION}

In the present study, quantitative confocal microscopic evaluation of NMDAR1 immunofluorescence intensity revealed that both estradiol and estradiol plus progesterone treatment in ovariectomized rats induced a significant intensity increase within the somata and dendrites of CA1 pyramidal cells in comparison with nonsteroid treated ovariectomized rats. A smaller, although statistically significant, increase was also observed within the somata of the dentate gyrus granule cells of steroid-treated animals in comparison with nontreated ovariectomized animals but not 
OVX
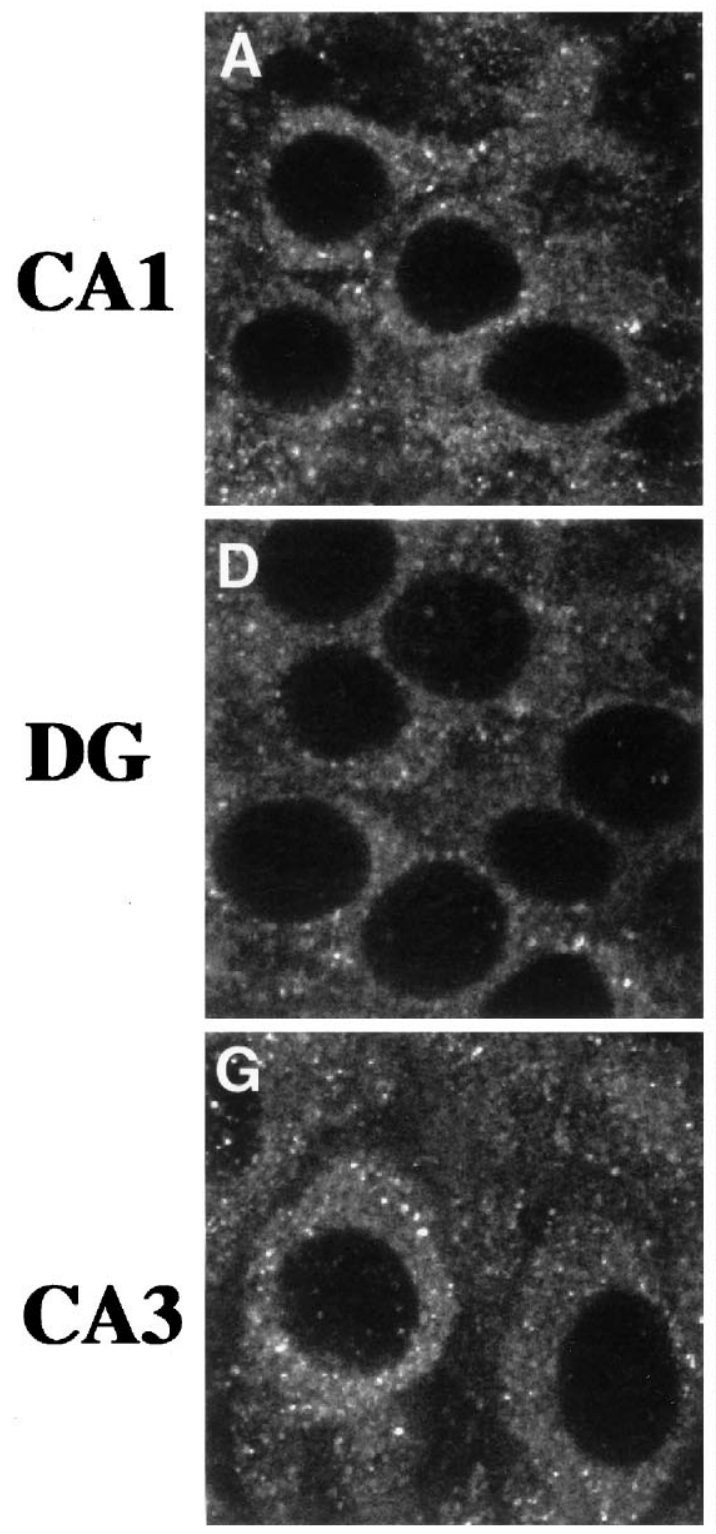

OVX+E
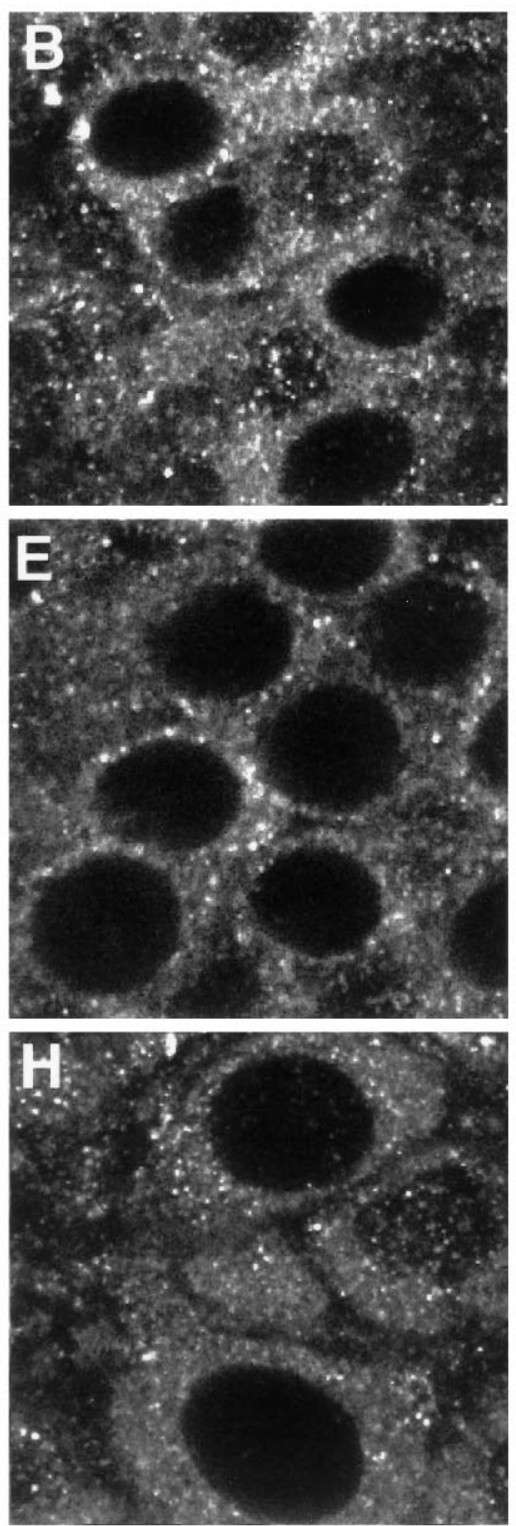
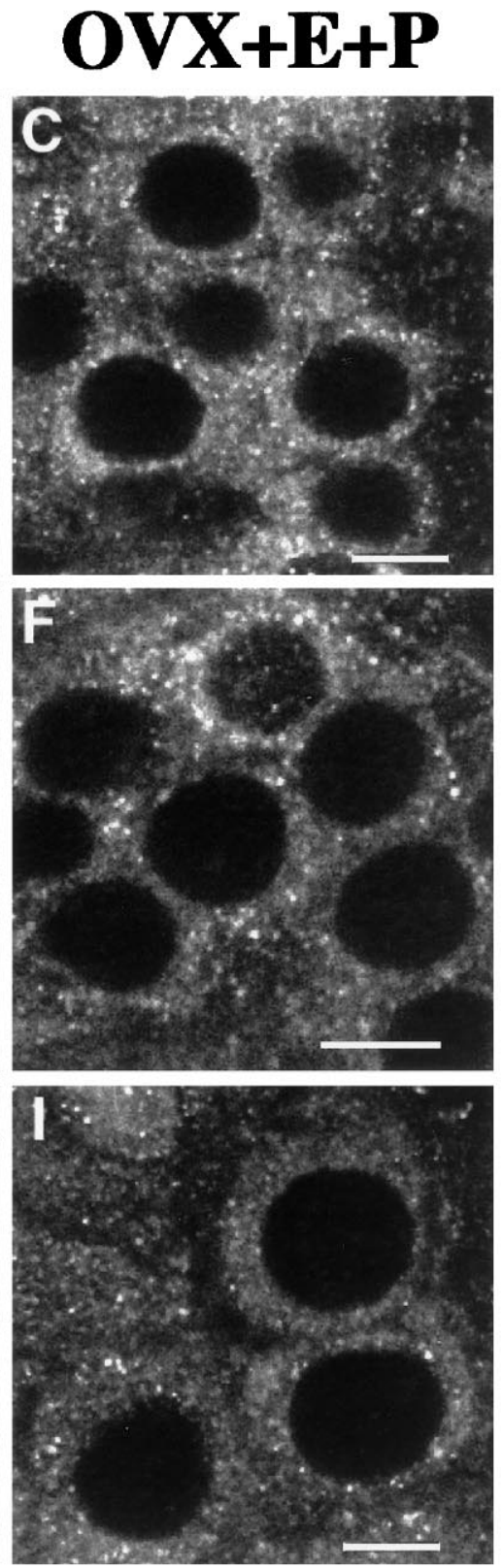

Figure 2. Examples of CLSM images of NMDAR1 immunolabeled somata in CA1 $(A-C)$, the dentate gyrus $(D-F)$, and CA3 $(G-I)$ of OVX $(A$, $D, G), \mathrm{OVX}+\mathrm{E}(B, E, H)$, and $\mathrm{OVX}+\mathrm{E}+\mathrm{P}(C, F, I)$ rats. Note the presence of punctate staining within the cytoplasm surrounding the unlabeled nuclei (see Discussion). When comparing the CA1 fields, an increase in the somatic intensity of staining is evident in the $\mathrm{OVX}+\mathrm{E}$ and $\mathrm{OVX}+\mathrm{E}+\mathrm{P}$ rats $(B, C)$ as compared with the OVX rats $(A)$. This increase in the steroid-treated ovariectomized rats is also apparent in the dentate gyrus $(E$, $F$ compared with $D)$, although to a lesser extent. There is no obvious difference in intensity levels among the three groups in the CA3 field $(G-I)$. $D G$, Dentate gyrus; $O V X$, ovariectomized rats; $O V X+E$, estradiol-treated ovariectomized rats; $O V X+E+P$, estradiol plus progesterone-treated ovariectomized rats. Scale bars, $10 \mu \mathrm{m}$.

within their dendrites. Because there was a trend toward an intensity increase in the granule cell dendrites, the absence of a significant change may have been the result of an inability to detect small intensity increases because of a degree of variability inherent in this technique. Additionally, there were no detectable differences in intensity levels within CA3 pyramidal cell somata of animals from all three groups. In situ hybridization analysis revealed no accompanying detectable alterations in NMDAR1 mRNA levels in any hippocampal subfield in steroid-treated rats as compared with OVX rats.

\section{Interpretation of NMDAR1 immunofluorescence intensity data}

The use of confocal microscopy yields high-resolution, crosssectional images of neurons, which when coupled with gray-level intensity quantification and a photometic offset, enabled us to obtain intensity measurements within major cellular compartments. Several factors contribute to the validity of the immunofluorescence intensity results as representing significant alterations between nontreated ovariectomized rats and steroidtreated ovariectomized rats. First, methodological variability was 

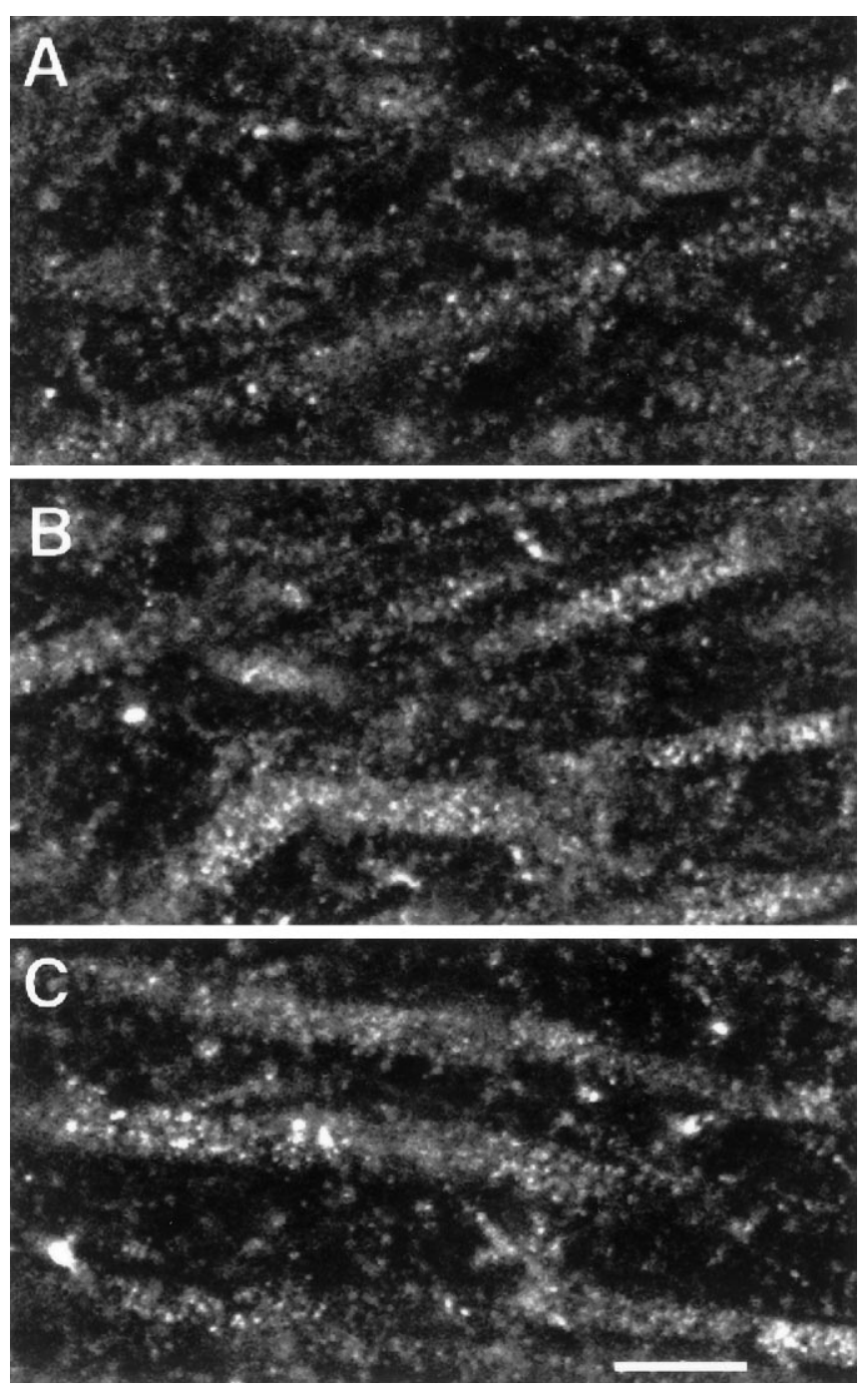

Figure 3. Examples of CLSM images of NMDAR1 immunolabeled dendrites in the CA1 subfield of an OVX rat $(A)$, an OVX $+\mathrm{E}$ rat $(B)$, and an $\mathrm{OVX}+\mathrm{E}+\mathrm{P}$ rat $(C)$. Note the presence of punctate staining within the cytoplasm of the dendritic segments (see Discussion) and the increased intensity of staining within the CA1 dendrites of the steroid-treated ovariectomized rats $(B, C)$ rats compared with the nonsteroid-treated ovariectomized rats $(A)$. Scale bar, $10 \mu \mathrm{m}$.

reduced by rigidly controlling tissue processing, immunofluorescent staining, confocal parameters, and analysis design. As a result, variability was minimized and intensity changes that were both consistent and of a substantial magnitude yielded statistically significant results. Additionally, the positive findings were regionally specific and occurred with greatest magnitude in CA1, the hippocampal field, in which estrogen-induced morphological changes have been observed (Gould et al., 1990; Woolley et al., 1990; Woolley and McEwen, 1992, 1994). Our results also correlate with an increased number of NMDAR agonist binding sites (Weiland, 1992) and a suggested functional increase in NMDARmediated synaptic activity (Wong and Moss, 1992) in the CA1 neurons after estradiol treatment. Lastly, no intensity differences were observed in any hippocampal field after an identical analysis with a monoclonal antibody to MAP2, arguing against a more general increase in protein production.

Immunoelectron microscopic descriptions of the ultrastructural distribution of the NMDAR1 subunit aids in the interpretation of
A
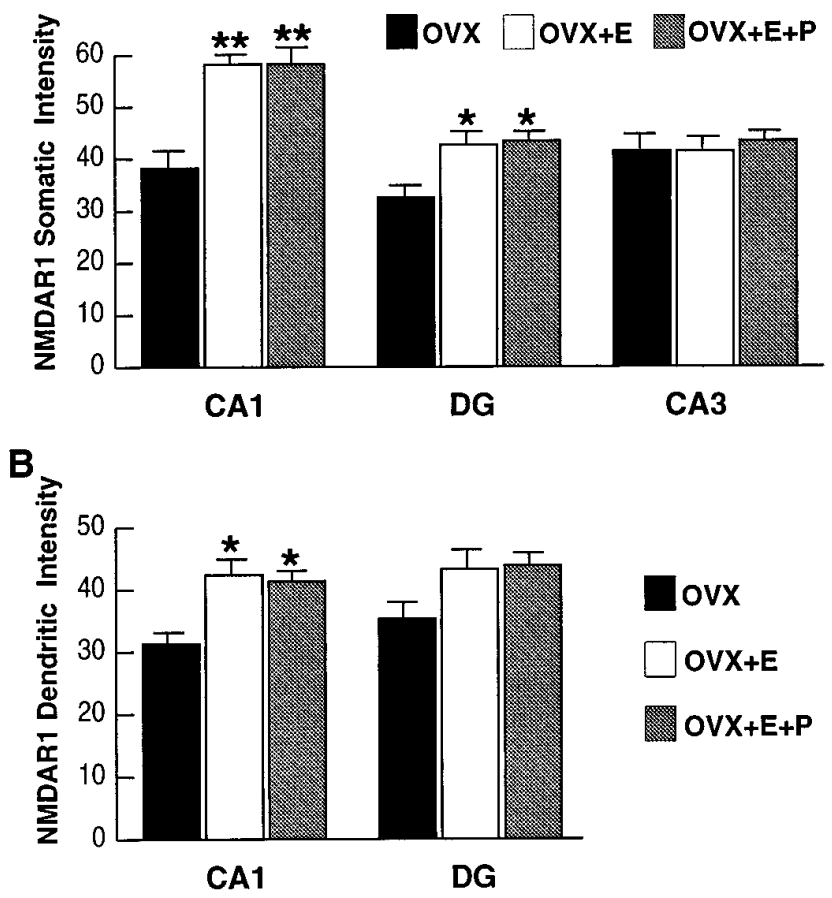

Figure 4. Bar graphs depicting NMDAR1 immunofluorescence intensity measurements in the somata $(A)$ and dendrites $(B)$ of the CA1, DG, and CA3 fields of the hippocampus. For somatic intensity measurements $(A)$, there is a significant increase in both CA1 and the dentate gyrus when comparing $\mathrm{OVX}+\mathrm{E}$ and $\mathrm{OVX}+\mathrm{E}+\mathrm{P}$ rats with $\mathrm{OVX}$ rats. Based on the results of the somatic intensity measurements $(A)$, the CA1 and the DG dendritic fields were quantified $(B)$. In $B$, note that there are significant increases only in the dendritic intensity measurements of steroid-treated rats as compared with the OVX rats in the CA1 subfield, although there is a trend toward increase in the dentate gyrus. Values represent mean \pm $\mathrm{SEM}$ for six OVX rats and five $\mathrm{OVX}+\mathrm{E}$ and $\mathrm{OVX}+\mathrm{E}+\mathrm{P}$ rats $(24$ measurements per rat). ${ }^{*} p<0.05,{ }^{* *} p<0.0001$ compared with OVX group; ANOVA and Sheffe's test.

our results. Similar to our qualitative CLSM observations, electron microscopic descriptions of rat hippocampal neurons revealed a patchy distribution of NMDAR1 immunocytochemical deposition throughout the somatodendritic cytoplasm (Petralia et al., 1994). In the somata and dendrites, these patches were associated with bundles of microtubules and the surface of mitochondria as well as with rough endoplasmic reticulum, golgi apparatus, and the nuclear envelope in somata (Petralia et al., 1994). The association of concentrations of NMDAR1 subunits with these subcellular structures suggests that the cytoplasmic patches represent the synthesis, processing, and transport pools of the protein. Previous CLSM investigations have demonstrated that immunoreactive intensity can reflect protein concentration (Good et al., 1992; Dodge et al., 1993), suggesting that our data may also represent alterations in protein concentration within these cytoplasmic pools. Given both an increase in NMDA agonist binding sites at the membrane (Weiland, 1992) and an increase in synaptic activity consistent with an enhancement of the NMDAR (Wong and Moss, 1992), alterations in the cytoplasmic pool of receptors appear to be reflected at the synaptic level.

\section{Mechanism of estrogen regulation of the NMDAR}

Consideration of both the immunofluorescence intensity and in situ hybridization data together suggests that alterations in NMDAR1 immunofluorescence are the result of post- 
OVX
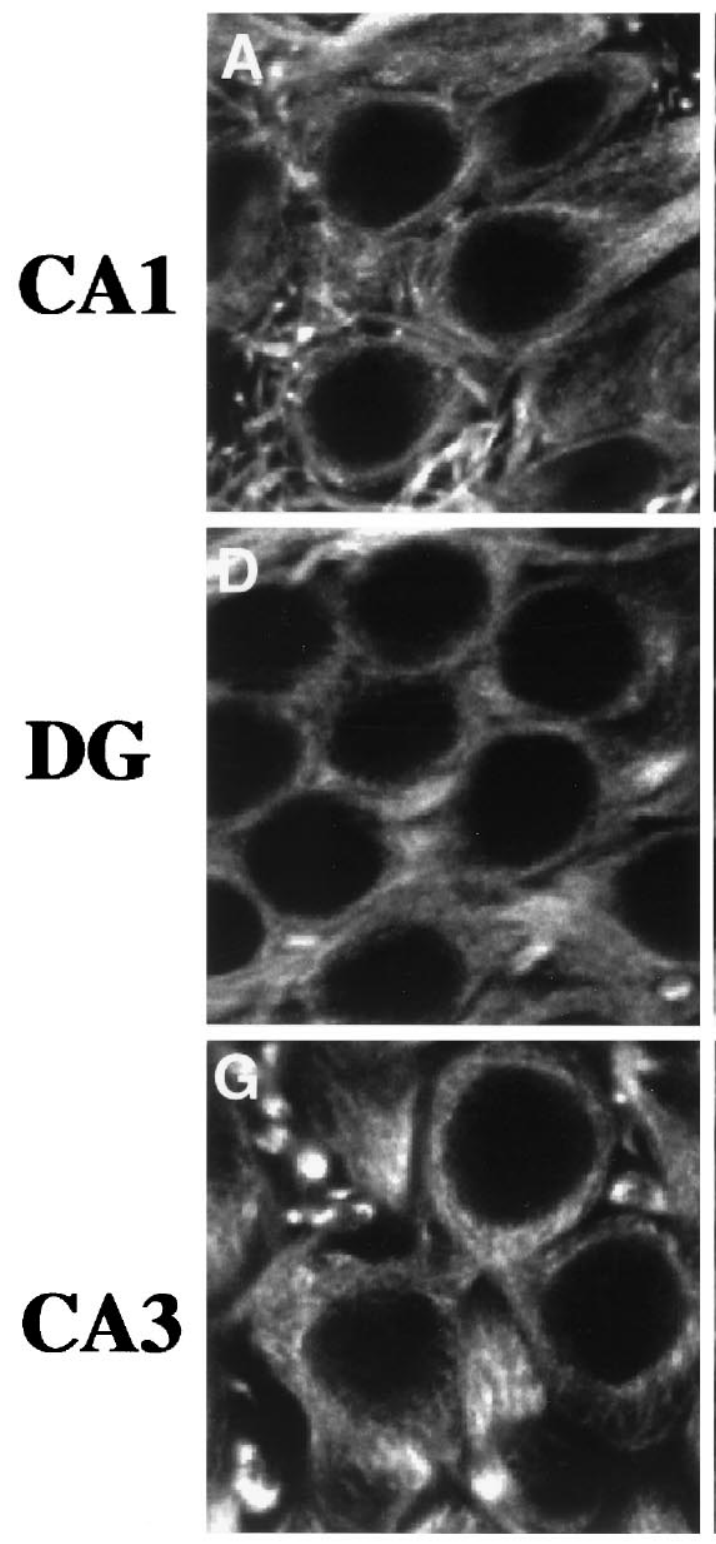

OVX+E
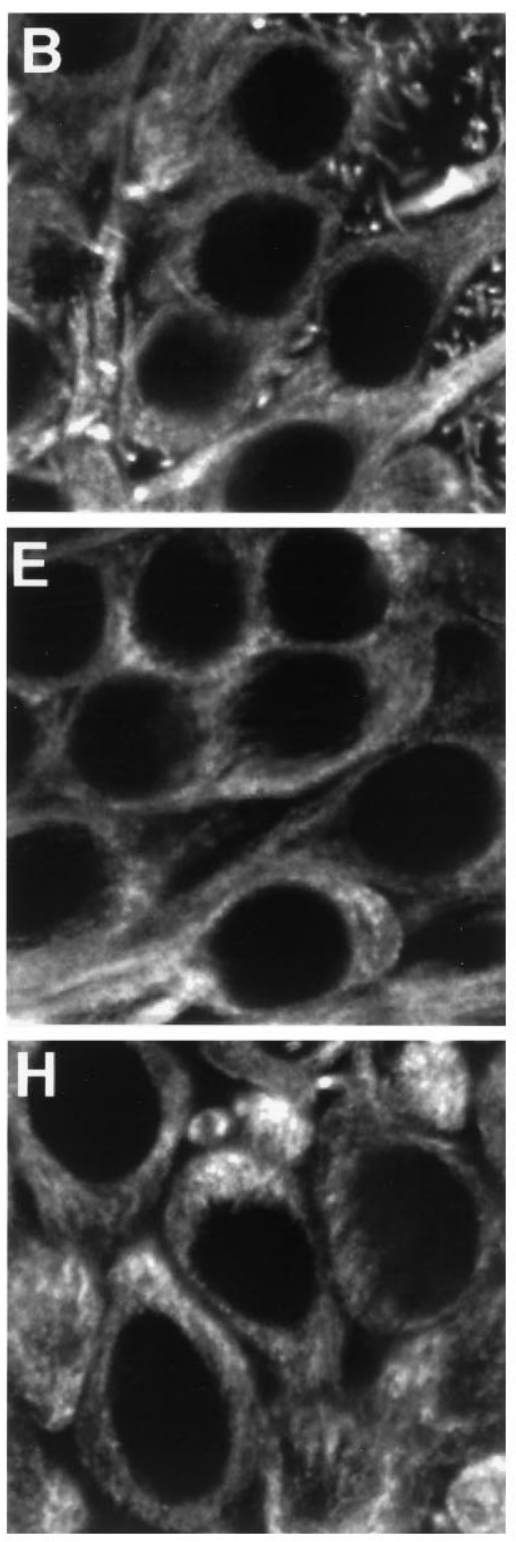

OVX+E+P
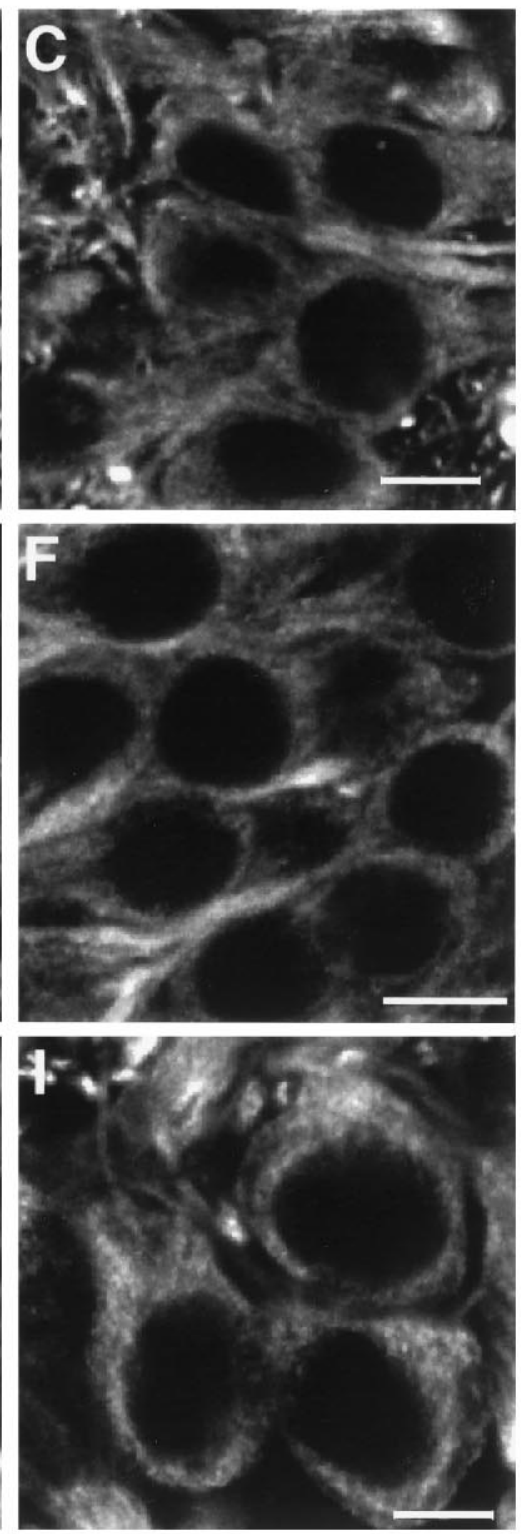

Figure 5. Examples of CLSM images of MAP2-immunolabeled somata in CA1 $(A-C)$, the dentate gyrus $(D-F)$, and CA3 $(G-I)$ of OVX rats $(A, D$, $G), \mathrm{OVX}+\mathrm{E}$ rats $(B, E, H)$, and $\mathrm{OVX}+\mathrm{E}+\mathrm{P}$ rats $(C, F, I)$. Note that there are no obvious differences in intensity levels when comparing the different experimental groups within any hippocampal subfield. Scale bars, $10 \mu \mathrm{m}$.

transcriptional regulation of the NMDAR1 protein. Reasonable possibilities of post-transcriptional regulation include an increase in rate of protein translation and/or post-translational modifications such as an alteration in the rate of protein degradation. Given the association of NMDAR1 protein with microtubules in the dendrites, intensity increases in the CA1 dendrites may be the result of increased dendritic transport. It is possible that the in situ hybridization technique is not sensitive enough to detect subtle changes in mRNA levels; however, post-transcriptional control of NMDAR1 protein expression has been demonstrated previously in PC12 cells, in which NMDAR1 mRNA is transcribed but not translated (Sucher et al., 1993). Additionally, two examples of post-transcriptional regulation of the NMDAR1 subunit have been recently documented. Both cultured cortical neurons that were chronically treated with an NMDAR antagonist (Follesa and Ticku, 1996) and the hippocampus of rats treated chronically with ethanol (Trevisan et al., 1994; Follesa and Ticku, 1995) exhibited increased levels of NMDAR1 protein with no detectable change in NMDAR1 mRNA levels.

The mechanism of regulation suggested by these results is somewhat different from the classical cellular mechanism of steroid hormone regulation of gene transcription. This is indicated by previous observations that suggest that estrogen regulation of NMDARs may be mediated by trans-synaptic interactions (Weiland, 1992; Woolley and McEwen, 1993; Woolley and McEwen, 1994). Indeed, putative intracellular estrogen receptors identified by autoradiography (Loy et al., 1988) and immunocytochemistry (Don Carlos et al., 1991; Weiland et al., 1996) are never found in 


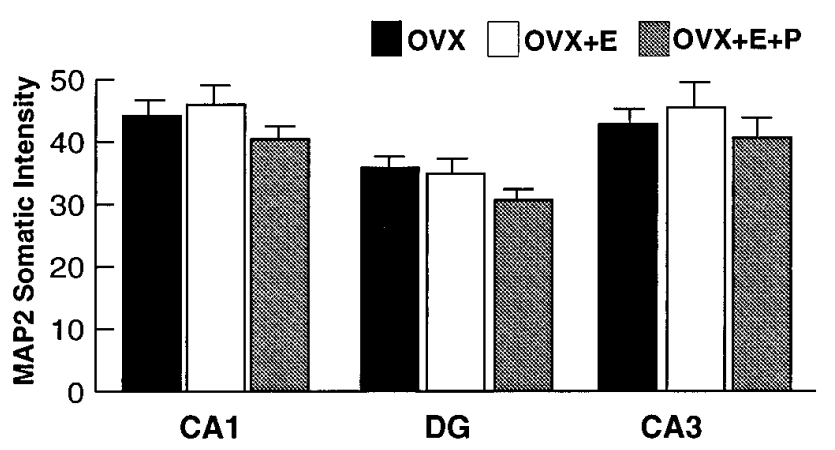

Figure 6. Bar graphs depicting MAP2 immunofluorescence intensity measurements in the somata of the CA1, dentate gyrus, and CA3 fields of the hippocampus. Quantitative analysis revealed no statistically significant differences among the three groups when comparing within a hippocampal field. Values represent mean \pm SEM for six OVX rats, five OVX + E rats, and $\mathrm{OVX}+\mathrm{E}+\mathrm{P}$ rats $(24$ measurements per rat).
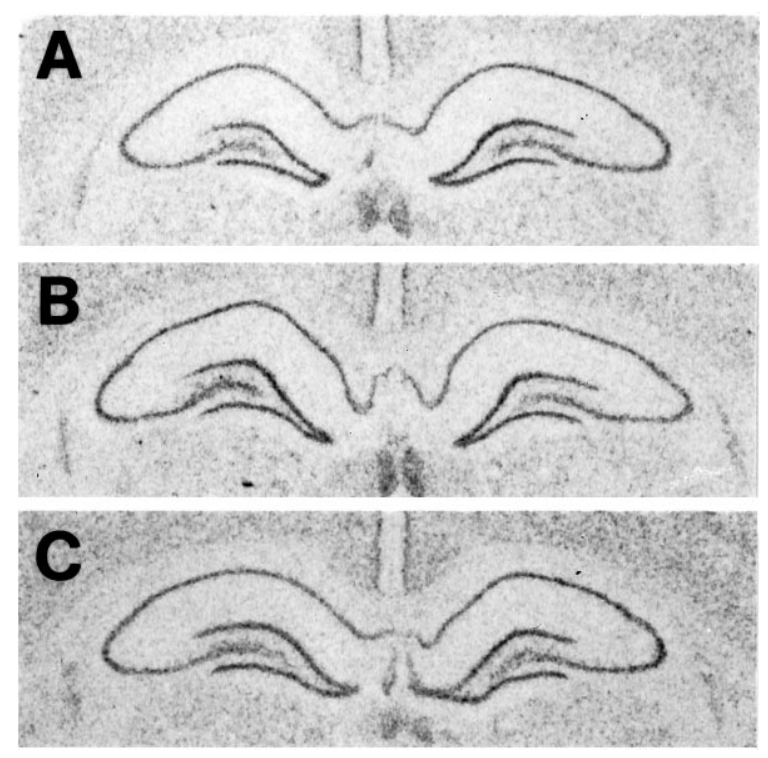

Figure 7. Photomicrographs of film autoradiograms show NMDAR1 mRNA hybridization in the hippocampus of an OVX rat $(A)$, an OVX $+\mathrm{E}$ rat $(B)$, and an $\mathrm{OVX}+\mathrm{E}+\mathrm{P}$ rat $(C)$. Note that there is no overt difference in hybridization distribution or intensity in the hippocampal subfields among rats from different treatment groups.

CA pyramidal neurons but rather are found in interneurons in the CA1, subiculum, and dentate gyrus subfields. Interestingly, very few such estrogen-sensitive interneurons are found in the CA3 region (Loy et al., 1988), in which we could find no estrogen effect on NMDAR1 immunofluorescence intensity in the present study. An estrogen-mediated increase in the expression of NMDARs and an increase in the density of excitatory spine synapses on CA1 pyramidal neurons that occur in an NMDA-dependent manner (Woolley and McEwen, 1994) thus may be reflections of the same underlying mechanism. This mechanism may involve a transsynaptic control of the excitability of pyramidal neurons via estrogen receptors in inhibitory interneurons, or it may reflect actions of estrogen more directly on the excitability of pyramidal neurons themselves by an as-yet undefined membrane mechanism. In either case, estrogen-induced changes in neuronal excitability may increase functional demands on the pyramidal neurons, leading to increased post-transcriptional expression of NMDAR1.

A more detailed characterization of estrogen-induced NMDAR

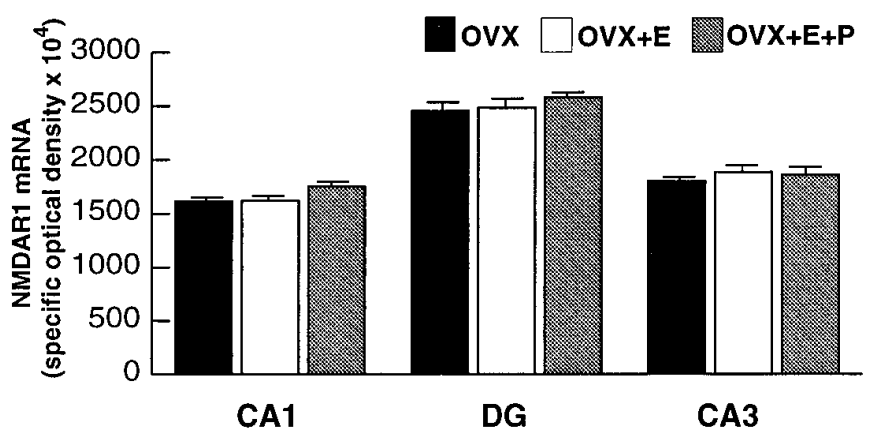

Figure 8. Bar graphs depicting the quantification of NMDAR1 mRNA labeling in the CA1, dentate gyrus, and CA3 subfields of the hippocampus. There were no statistically significant differences in optical density measurements in any of the subfields among the three groups. Values represent mean \pm SEM for nine OVX rats, nine OVX $+E$, and nine $\mathrm{OVX}+\mathrm{E}+\mathrm{P}$ rats.

protein regulation remains to be elucidated. The subcellular distribution of the NMDAR1 subunit has been demonstrated to be controlled by specific amino acid sequences that are located within a C-terminal exon that is subject to alternative splicing (Ehlers et al., 1995). The antibody used in this study detects all NMDAR1 splice variants. Future studies using splice variantspecific antibodies are necessary to determine whether estrogeninduced regulation is NMDAR1 splice variant-specific. Additionally, a possible stoichiometric change in the NMDAR complex in CA1 is suggested by disparate results obtained using different agonist versus antagonist NMDAR binding ligands (Weiland, 1992). A stoichiometric change may also account for our determination of a subtle intensity increase in the dentate gyrus, whereas autoradiographic analysis revealed no change in NMDA agonist binding and a sight decrease in NMDA noncompetitive antagonist binding in the dentate gyrus (Weiland, 1992). The NMDAR is a heteromeric complex of several subunits, most likely consisting of an NMDAR1 subunit and one or more of four different NMDAR2 subunits that affect function and ligand binding characteristics (Monyer et al., 1992; Hollmann and Heinemann, 1994). Differential regulation of NMDAR subunits will be investigated after the development of antibodies specific for NMDAR2 subunits.

\section{Functional significance}

Estradiol has an important role in cognitive function in experimental animals (Luine, 1994; Singh et al., 1994) and in maintaining certain memory functions in surgically postmenopausal women given estradiol replacement therapy, which are compromised in women not given estradiol (Philips and Sherwin, 1992a). Additionally, in some women, specific memory functions were found to co-vary with sex steroid plasma concentrations across the menstrual cycle (Philips and Sherwin, 1992b). Considering the well-established role of the hippocampus (Zola et al., 1986; Alvarez et al., 1995) and NMDARs (Morris et al., 1982; Bliss and Collingridge, 1993) in learning and memory formation, estrogen may affect memory by maintaining dendritic spines (Gould et al., 1990), excitatory synapses (Woolley and McEwen, 1992), and NMDARs in specific hippocampal neuronal populations via mechanisms suggested in this study. We have previously characterized an intradendritic alteration in NMDAR1 immunofluorescence intensity within the dentate gyrus of aged female monkeys that revealed a decrease in intensity within dendritic segments of the outer 
molecular layer relative to dendritic segments of the inner molecular layer (Gazzaley et al., 1996a). Given our present findings of estrogen's role in regulating NMDAR1 immunofluorescence in the dentate gyrus after ovariectomy, it is possible that an age-related estrogen decrease in the aged female monkeys may have contributed to the NMDAR1 alterations observed previously.

In addition to increasing the duration of EPSPs in CA1 neurons in a manner suggestive of NMDAR enhancement, estrogen treatment also induced repetitive firing in some CA1 neurons that resembled epileptic bursting responses (Wong and Moss, 1992). These findings are consistent with other studies that revealed that estradiol replacement facilitates the induction of kindled seizures (Buterbaugh and Hudson, 1991) and decreases the threshold for seizure induction (Terasawa and Timiras, 1968) in the hippocampus of ovariectomized rats. Additionally, an increased incidence of seizures was observed in women with catamenial epilepsy that correlated with fluctuations in estrogen levels across the menstrual cycle (Backstrom, 1976). Based on the NMDAR's important role in seizure induction in experimental animals (Gilbert, 1988; Sato et al., 1989), estrogen may induce seizure activity by regulation of the NMDAR via modification of the NMDAR1 protein. Although there is no direct evidence for the link between estrogeninduced NMDAR regulation and behavioral changes, it is reasonable that post-transcriptional NMDAR1 regulation has functional implications for learning and memory formation, age-related cognitive decline, and seizure induction.

\section{REFERENCES}

Alvarez P, Zola MS, Squire LR (1995) Damage limited to the hippocampal region produces long-lasting memory impairment in monkeys. J Neurosci 15:3796-3807.

Backstrom T (1976) Epileptic seizures in women related to plasma estrogen and progesterone during the menstrual cycle. Acta Neurol Scand 54:321-347.

Bliss TVP, Collingridge GL (1993) A synaptic model of memory: longterm potentiation in the hippocampus. Nature 361:31-39.

Buterbaugh GC, Hudson GM (1991) Estradiol replacement to female rats facilitates dorsal hippocampal but not ventral hippocampal kindled seizure acquisition. Exp Neurol 111:55-64.

Dodge DE, Rucker RB, Singh G, Plopper CG (1993) Quantitative comparison of intracellular concentration and volume of Clara cell $10 \mathrm{KD}$ protein in rat bronchi and bronchioles based in laser scanning confocal microscopy. J Histochem Cytochem 41:1171-1183.

Don Carlos LL, Monroy E, Morrell J (1991) Distribution of estrogen receptor-immunoreactive cells in the forebrain of the female guinea pig. J Comp Neurol 305:591-612.

Ehlers MD, Tingley WG, Huganir RL (1995) Regulated subcellular distribution of the NR1 subunit of the NMDA receptor. Science 269:1734-1737.

Follesa P, Ticku MK (1995) Chronic ethanol treatment differentially regulates NMDA receptor subunit mRNA expression in rat brain. Mol Brain Res 29:99-106.

Follesa P, Ticku MK (1996) NMDA receptor upregulation: molecular studies in cultured mouse cortical neurons after chronic antagonist exposure. J Neurosci 16:2172-2178.

Gazzaley AH, Siegel SJ, Kordower JH, Mufson EJ, Morrison JH (1996a) Circuit-specific alterations of $N$-methyl-D-aspartate receptor subunit 1 in the dentate gyrus of aged monkeys. Proc Natl Acad Sci USA 93:3121-3125.

Gazzaley AH, Benson DL, Huntley GW, Morrison JH (1996b) Laminaspecific regulation of NMDAR1 immunoreactivity in dentate gyrus following entorhinal cortex lesions. Soc Neurosci Abstr, in press.

Gilbert ME (1988) The NMDA-receptor antagonist, MK-801, suppresses limbic kindling and kindled seizures. Brain Res 463:90-99.

Good MJ, Hage WJ, Mummery CL, De Latt SW, Boonstra J (1992) Localization and quantification of epidermal growth factor receptors on single cells by confocal laser scanning microscopy. J Histochem Cytochem 40:1353-1361.

Gould E, Woolley CS, Frankfurt M, McEwen BS (1990) Gonadal steroids regulate dendritic spine density in hippocampal pyramidal cells in adulthood. J Neurosci 4:1286-1291.

Hollmann M, Heinemann S (1994) Cloned glutamate receptors. Annu Rev Neurosci 17:31-108.

Huber G, Matus A (1984) Differences in the cellular distributions of two microtubule-associated proteins, MAP1 and MAP2, in rat brain. J Neurosci 4:151-160.

Loy R, Gerlach JL, McEwen BS (1988) Autoradiographic localization of estradiol-binding neurons in the rat hippocampal formation and entorhinal cortex. Dev Brain Res 39:245-251.

Luine VN (1994) Steroid hormone influences on spatial memory. Ann NY Acad Sci 14:201-211.

McEwen BS, Gould E, Orchinik M, Weiland NG, Woolley CS (1995) Oestrogens and the structural and functional plasticity of neurons: implications for memory, ageing and neurodegenerative processes. In: Non-reproductive actions of sex steroids (Ciba Foundation Symposium) (Bock GR, Goode JA, eds), pp 52-73. Chichester: Wiley.

Monyer H, Sprengel R, Schoepfer R, Herb A, Higuchi M, Lomeli H, Burnashev N, Sakmann B, Seeburg PH (1992) Heteromeric NMDA receptors: Molecular and functional distinction of subtypes. Science 256:1271-1221.

Moriyoshi K, Masu M, Takahiro I, Shigemoto R, Mizuno N, Nakanishi S (1991) Molecular cloning and characterization of the rat NMDA receptor. Nature 354:31-37.

Morris RGM, Garrud P, Rawlins JNP, O'Keefe J (1982) Placenaviagation in rats with hippocampal lesions. Nature 297:681-683.

Morris RGM, Anderson E, Lynch GS, Baudry M (1986) Selective impairment of learning and blockade of long-term potentiation by an $N$-methyl-D-aspartate receptor antagonist, AP5. Nature 319:774-776.

Nakanishi S (1992) Molecular diversity of glutamate receptors and implications for brain function. Science 258:597-603.

Orchinik M, Weiland NG, McEwen BS (1994) Adrenalectomy selectively regulates GABAA receptor subunit expression in the hippocampus. Mol Cell Neurosci 5:451-458.

Petralia RS, Yokotani N, Wenthold RJ (1994) Light and electron microscope distribution of the NMDA receptor subunit NMDAR1 in the rat nervous system using a selective anti-peptide antibody. J Neurosci 14:667-696.

Philips SM, Sherwin BB (1992a) Effects of estrogen on memory function in surgically menopausal women. Psychoneuroendocrinology 17:485-495.

Philips SM, Sherwin BB (1992b) Variations in memory function and sex steroid hormones across the menstrual cycle. Psychoneuroendocrinology 17:497-506.

Sato K, Morimot K, Hiramatsu M, Mori A, Otsuki S (1989) Effect of a noncompetitive antagonist (MK-801) of NMDA receptors on convulsions and brain amino acid level in E1 mice. Neurochem Res 14:741-744.

Siegel SJ, Janssen WG, Gasic GP, Jahn R, Heinemann SF, Morrison JH (1994) Regional, cellular and ultrastructural distribution of $N$-methylD-aspartate receptor subunit 1 in monkey hippocampus. Proc Natl Acad Sci USA 91:564-568.

Singh M, Meyer EM, Millard WJ, Simpkins JW (1994) Ovarian steroid deprivation results in reversible learning impairment and compromised cholinergic function in female Sprague-Dawley rats. Brain Res 644:305-312.

Sucher NJ, Brose N, Dietcher DL, Awobuluyi M, Gassic GP, Bading H, Cepko CL, Greenberg ME, Jahn R, Heinemann SF, Lipton SA (1993) Expression of endogenous NMDAR1 transcripts without receptor protein suggests post-transcriptional control in PC12 cells. J Biol Chem 30:22299-22304

Terasawa E, Timiras PS (1968) Electrical activity during the estrous cycle of the rat: cyclic changes in limbic structures. Endocrinology 83:203-216.

Trevisan L, Fitzgerald LW, Brose N, Gasic GP, Heinemann SF, Duman RS, Nestler EJ (1994) Chronic ingestion of ethanol up-regulates NMDAR1 receptor subunit immunoreactivity in rat hippocampus. J Neurochem 62:1635-1638.

Weiland NG (1992) Estradiol selectively regulates agonist binding sites on the $N$-methyl-D-aspartate receptor complex in the CA1 region of the hippocampus. Endocrinology 131:662-668. 
Weiland NG, Orchinik M (1995) Specific subunit mRNAs of the GABAA receptor are regulated by progesterone in subfields of the hippocampus. Mol Brain Res 32:271-278.

Weiland NG, Orikasa C, Hayashi S, McEwen BS (1996) Localization of estrogen receptors in the hippocampus of male and female rats. Soc Neurosci Abstr, in press.

Wong M, Moss RL (1992) Long-term and short-term electrophysiological effects of estrogen on the synaptic properties of hippocampal CA1 neurons. J Neurosci 12:3217-3225.

Woolley CS, McEwen BS (1992) Estradiol mediates fluctuations in hippocampal synapse density during the estrous cycle in the adult rat. J Neurosci 12:2549-2554.
Woolley CS, McEwen BS (1993) Roles of estradiol and progesterone in regulation of hippocampal dendritic spine density during the estrous cycle in the rat. J Comp Neurol 336:293-306.

Woolley CS, McEwen BS (1994) Estradiol regulates hippocampal dendritic spine density via and $N$-methyl-D-aspartate receptor-dependent mechanism. J Neurosci 14:7680-7687.

Woolley CW, Gould E, Frankfurt M, McEwen BS (1990) Naturally occurring fluctuations in dendritic spine density on adult hippocampal pyramidal neurons. J Neurosci 10:4035-4039.

Zola MS, Squire LR, Amaral DG (1986) Human amnesia and the medial temporal lobe region: enduring memory impairment following a bilateral lesion limited to the field CA1 of the hippocampus. J Neurosci 6:2950-2967. 